\title{
Thermodynamics of Moving Bodies or a New Approach to Emergent Gravity
}

\author{
Yormahmad Kholov \\ Department of Transport, Tajik Technical University, Dushanbe, Tajikistan
}

Email address:

yormahmad@gmail.com

\section{To cite this article:}

Yormahmad Kholov. Thermodynamics of Moving Bodies or a New Approach to Emergent Gravity. American Journal of Modern Physics. Vol. 9, No. 2, 2020, pp. 28-40. doi: 10.11648/j.ajmp.20200902.12

Received: May 24, 2020; Accepted: June 15, 2020; Published: June 28, 2020

\begin{abstract}
Recent theoretical developments propose that gravity is emergent phenomenon. In line with this, in this paper, we show that gravitational and inertial properties of matter can be sufficiently explained by thermodynamics for a system consisting of material systems immersed in the quantum vacuum energy reservoir without reference to the microscopic constituencies of the quantum vacuum. The study focuses on the transfer of energy and matter in the interaction of material system with its vacuum surroundings and the relation of those to the system's macroscopic state variables and mechanical behavior of the system associated with forces acting on it. This analysis suggests that vacuum energy density about material systems is diminished and quantum vacuum energy density field takes on specific gradient there. Hence, gravity appears as effect of the change in the energy density of medium related with presence of another material object modifying vacuum surroundings and causing spontaneous motion of the system to minimize its energy driven by the second law. Whereas, inertia is explained to be an emergent thermodynamic effect of the change in the vacuum energy field about objects associated with the alteration of energy of systems itself due to the transfer of energy between systems to its surroundings. When those energy transfer operations do not comply with the second law of thermodynamic in terms of direction and rate of the energy flow, there will be generated resistance to the imposed changes known as inertial force. In such representation the equivalence principle finds unique definition revealing its origin.
\end{abstract}

Keywords: Models of Quantum Gravity, Thermodynamics, Quantum Vacuum energy Density, Entropic Gravity, Inertia

\section{Introduction}

The connection between gravity and the principles of thermodynamics became obvious with the early works of Bekenshten and Hawking in the 1970s on black holes [1, 2]. These works have established the proportionality of a black hole's entropy to its horizon area and its temperature to the surface gravity at the black hole horizon. Another work has shown that the first law of thermodynamics describes relation of the entropy, temperature and the mass of the black hole [3].

The similarity of the formulation of laws of thermodynamics (LTD) and of gravitation was noted even before but it was left without deeper research. Only late in the 1990s were the general relativity's field equations derived by Jacobson using general thermodynamic considerations combined with the equivalence principle [4]. In this way, he concludes that the Einstein equation can be considered as an equation of the state of spacetime.
Investigating the relation between the first law of thermodynamics and the gravitational field equation for black hole spacetime Padmanabhan [5] inferred that Einstein's equation is a thermodynamic identity. His work motivated the appearance of number other works in this topic that confirmed the growing interest of physicists to this field. For more references on this topic see [6].

In the first decade of this century, Eric Verlinde [7] presented a model that explains gravity as an entropic force that is a consequence of the change of information related with the position of matter objects in space. His methodology is unique and unites the thermodynamic approach to gravity with the holographic principle. He concludes that gravity is not a fundamental interaction, but rather is an emergent phenomenon, which arises from the microscopic statistical behavior of matter as defined by the holographic principle.

In fact, Verlinde is not the first physicist to regard gravity as a non-fundamental interaction. One of the prominent ideas 
in this respect is the concept of induced gravity that proposed by Sakharov in 1968 [8]. According to his conjecture gravity is not a fundamental force but it is an induced effect associated with zero-point fluctuations of the vacuum.

Verlinde further extended his research and in 2011 presented a new work that attempted to give an explanation for the questioning rotation speed of stars in outskirts of galaxies without invoking the dark matter [9]. Verlinde's papers drew diverse responses from the scientific community as well as attracting a large amount of media exposure.

This entropic gravity concept has also motivated the publication of a number of new follow-up works in cosmology $[10,11]$ presenting a new viewpoints on the existing models, such as the derivation of the Friedman equations [12], thereby suggesting an explanation for the accelerated expansion rate of the Universe [13-15].

Some work from most recent studies shows Newtonian classical gravity in view of non-equilibrium thermodynamics via introducing an unspecified scalar field potential and analyzes the entropy production rate to explore the effects of irreversibility on the field [16].

A modern group of scientists unified by the idea of application of stochastics to quantum mechanics (tiled stochastic quantum mechanics) attempt to explain gravity as effect of matter interaction with granular quantum vacuum. Such, Caligiuri and Sorli, in their works consider that mass of elementary particle [17] and gravitation [18] have their origin in the diminished energy density of a quantum vacuum. Although this work obtained similar with our work result their reasoning for this proposal has non-thermodynamic basis.

Our aim in this paper is to show that gravity and inertia are emergent phenomena by use of another approach. Verlinde's approach shows that gravity is an entropic force and that it is a consequence of the change of information related with the position of matter objects in space. On the other hand, this change of information, entropy shall be linked with the change in energy of the system. This means that the emergence of gravity can equally be shown through an approach that is based on analyses of the changes in the energy of medium.

Analogy between Gravity and thermodynamics (TD) and the universality of both suggests that emergence of gravity and consequently inertia shall be explained by general principles of TD. Whole space in the universe filled with vacuum energy of huge density that encompasses all matter that can be assumed as an energy reservoir for material objects considered as thermodynamic systems. This fundamental medium is regarded as a real relativistically invariant quantum scene with its energy. Apparently, the interaction between matter and this energy filling whole space shall be the key in the determination of behavior of matter. In this setup, the exchange of energy associated with that interaction shall be governed by the laws of TD and the key conception to derive gravity and inertia will be energy. That is the changes in energy of matter and quantum vacuum, the energy transfers between both associated with relative relocation of matter objects and their acceleration in such surroundings. We will show that presence of a material object in vacuum causes the vacuum modification due to the vacuum energy alteration in the local surroundings that creates anisotropy and provides possibility to other objects in the vicinity to minimize their energy via relocation. Free or forced relocations (in such surroundings) and related accelerations cause energy transfers between objects and their vacuum surroundings. When the rate and direction of these energy transfers do not comply with $2^{\text {nd }}$ law of thermodynamics (2 LTD) there is generated a force, which appears as mechanical effects known to us as gravity and inertia. The origin of gravity and inertia therefore lies in the tendency of material objects to minimize their energy while they are viewed as thermodynamic systems immersed in the vacuum $^{1}$ energy reservoir with local anisotropy (due to locally altered vacuum energy density).

In this approach we apply the following assumptions. Although we used to the assumption that in any mechanical process energy from a system performing work is ultimately dissipated to the environment, the inverse case, however, assuming that energy from surrounding space is transferred to the system when work performed on it, is not well-accepted. For instance, while decelerating an object its kinetic energy is used to say that is dissipated to environment as heat (or as work), but for the acceleration case we conventionally used to say that energy to the system imparted from an external agent i.e. other objects, while it is more reasonable to consider that (kinetic) energy of the system is increased on the account of energy of environment by work done by external agent on the system. In other words the work done on the system by external agent (to speed it up) is used to transfer energy from surroundings to the system to increase its kinetic energy. In this context term surrounding implies surrounding vacuum. This statement is in the spirit of TD since it implies that the system is in constant interaction with its surroundings as in the deceleration case and therefore it is more consistent approach. This will be the important assumption in this work.

Thus, in this paper we present a model that explains emergence of gravity and inertia based on the analysis of changes in the energy of a system and its surroundings that abide by laws of thermodynamics. The study involves an investigation of energy exchange between a material object and the quantum vacuum energy reservoir with finite energy density.

The second LTD in its known formulation affirming that heat spontaneously moves from hot to cold place establishes connection between energy transfer and the gradient in the background energy field. Application of this principle to a mass immersed in vacuum by virtue of the mass-energy equivalence suggests that motion of mass can also be consequence of the alterations of energy in surroundings.

\footnotetext{
${ }^{1}$ Far back in 1907 Max Planck [16] legitimized application of the basic laws of mechanics and the two laws of thermodynamics to «a system devoid of ponderable matter» comprised of electromagnetic radiation, which today is known as electromagnetic quantum vacuum.
} 
One of the bright examples to investigate this conception would be gravitational freefall.

The physical fact that gravitational freefall motion of matter is due to the second law makes thermodynamics a theory that can play a key role in the study of gravitation and motion. This point relates motion with thermodynamics and requires particular attention as it can provide deeper insight into understanding the nature of gravity and inertia.

It is acknowledged that an object falling in the gravitational field minimizes its energy state, thus showing its compliance with the second law of thermodynamics. In this setting, if we assume that the object is a (closed) thermodynamic system that resides in the quantum vacuum with huge energy density filling the whole space and comprising the thermodynamic surroundings for that system, then from the system's behavior to minimize its energy state (in the gravitational field) involving spontaneous tendentious (freefall) motion, it can be inferred that the surroundings, i.e. the vacuum in gravitational field has a specific, an energy density gradient. That is, in any point of such surroundings a free object undertakes spontaneous motion to an adjacent point in the direction of the source of the field (where surroundings' energy has less density) in order to minimize its energy through converting it to kinetic energy of motion, which is eventually subject to transfer to its surroundings as work (or as heat). This behavior illustrates that vacuum energy (density) field in the object's surroundings is anisotropic or more specifically, energy in the locale where the object moves to is lesser than in the initial point.

Such, although pursuing the same goal as Verlinde's proposal this work does not invoke quantum information theories. Instead, it connects gravity and inertia with the energy of quantum vacuum fields using thermodynamic principles. In this regard it is a unique approach and has as yet undiscovered potential.

\section{Emergent Gravity}

Imagine an object placed in point 1 of a gravitational field of an adjacent massive body; let's fix it relative to the body, thereby maintaining its equilibrium. Consider that the object is freed $^{2}$ and undertakes spontaneous freefall motion to an adjacent point 2 in the direction of the source of the field along axis, $r$, which connects the centers of mass of both objects, so that $\Delta r=\left(r_{2}-r_{1}\right) \rightarrow 0$. Hereby potential energy of the object is decreased for $\Delta E$, at the same time kinetic energy of it is increased for the same magnitude. Let the motion of the object to be halted by an obstacle at point 2; this forces the object to perform work on the obstacle and thereby transfer energy $\Delta E$ as work to the surroundings and find new equilibrium with surroundings.

We consider the object as a closed thermodynamic system with total energy $E_{l}$ (at point 1 ) that is in equilibrium with its surroundings, i.e. vacuum with energy density, $\rho_{v l}$ in this locale. At point 2 energy of the system is $E_{2}$ and vacuum

2 This is considered as a thermodynamic operation performed on the system energy density is $\rho_{v 2}$. Let us find correlation between the change in energy of the system and energy of its surroundings. If the system at point 1 has energy $E_{l}$ and be at equilibrium with its surroundings - vacuum with energy density $\rho_{v l}$ and by spontaneous process of freefalling minimizing its energy level to $E_{2}\left(E_{2}<E_{l}\right)$ at adjacent point 2 where the system finds new equilibrium with its surroundings. Then, assuming that the reaction forces for maintaining equilibrium (object in rest at point 1 and 2) of the system in 1 and 2 have equal magnitude (since $\Delta r \rightarrow 0$ ), it is logical to conclude that surrounding vacuum at the last point has energy density $\rho_{v 2}$, which is proportional to energy of the system in the point, $E_{2}$ :

$$
E_{1} / E_{2}=\rho_{v 1} / \rho_{v 2}
$$

In freefall motion from point 1 to 2 the system's potential energy, as recognized, decreases while its kinetic energy increases. However, if we compare the system's energy in rest state in both locations then we see that total energy of the system has decreased (in point 2) since kinetic energy that the system generates, in fact, dissipates or transferred to surrounding as heat or work when the system brought to rest state. Therefore we can qualify kinetic energy as an active form of energy that the system has to discharge to surroundings while spontaneously relocates to the locale, where surroundings' energy has less density.

Such, in its freefall motion in gravitational field a system releases to surrounding an amount of energy proportional to the change of the energy density of surroundings between earlier and new locale, due to the second law. Consequently, energy of the system in the field is defined by the energy density distribution in the local surroundings.

Then from the above, we can deduce that the difference in energy of the freefalling system displaced for $\Delta r$ (and being in equilibrium state earlier and after the displacement) is proportional to the rate of the change in surroundings' energy density per distance, i.e. the change in background vacuum energy, that is

$$
\Delta E_{k} \propto \Delta \rho_{v} / \Delta r,\left(\text { or } \Delta E=-\Delta E_{k} \propto \Delta \rho_{v} / \Delta r\right)
$$

where, $\Delta E=E_{2}-E_{1}$ is the change in energy of the system between points 2 and $1, \Delta E_{k}$ is the change in kinetic energy of the system and $\Delta \rho_{v}=\rho_{v 2}-\rho_{v 1}$ - is the change in the energy density of the vacuum between respective points. The negative sign indicates that $\Delta E_{k}$ increases in the direction of $\rho_{v}$ decrease. Expression (2) can therefore be rewritten in this form:

$$
\Delta E_{k} \propto-\operatorname{grad} \rho_{v}
$$

The above indicates the availability of the vacuum energy density gradient in the local area, which is a cause of spatial anisotropy in the field, determines magnitude of the energy to be transferred to surroundings from the system. It is equal to change in kinetic energy of the system (in the given frame of reference).

On the other hand, the above implies that the change in energy density of vacuum for $-\Delta \rho_{v}$ along elementary 
displacement $\Delta r$ defines the change in energy of the system, $\Delta E$, which is equal to the work done by gravitational field on the system $^{3}$ (in conventional representation) that can be formulated this way:

$$
\Delta E / m=-\Delta \rho_{v}
$$

where, $m$ is volumetric mass of the material system and it is introduced to make both sides of the equation the same physical magnitude, i.e. energy density. If we here consider classical formulation of gravitational potential: $\Delta E=m g \Delta r$, then (4) yields:

$$
\bar{g}=-\Delta \rho_{v} / \Delta r \text { or } \bar{g}=-\operatorname{grad} \rho_{\mathrm{v}}
$$

The latest derivation confirms that acceleration of the system in freefall (inertial motion) defined by the gradient of energy density of its surroundings, i.e. vacuum. Acceleration behavior of a system in freefall (as a pure inertial motion) defined by energy pattern of surroundings triggering tendency of matter minimize its energy attributable to the second law. Or, that is freefall acceleration of an object is entirely defined by the local vacuum energy density gradient about the object.

Furthermore, the above consideration (leading to expression (4 and initially 1) implies that energy (the negative potential) provided to the system is necessary to escape "bound energy"- associated with $\Delta \rho_{v}$ corresponding to elementary translation $\Delta r$.

Thus, a force thereby arises when an action attempts to prevent an object with mass, $m$, from performance of the spontaneous (freefall) motion, which is driven by the second law. Let us formulate this force.

Then in view of the above and expression (5) Newton's second law can be formulated in the following way:

$$
\bar{F}=m \bar{g}=-m \nabla \rho_{\mathrm{v}},
$$

where, $\nabla \rho_{v}$ is the gradient of energy density of vacuum about a particle with mass $m$. For multi-particle material object, assuming that $\nabla \rho_{v}$ has different magnitude across the body volume, resulting force will be as a sum of all elementary forces for constituencies of the body, i.e. $\bar{F}=-\sum m_{i} \nabla \rho_{v i}$. Formulation of the gravitational force might be obtained through calculation of radiation pressure for constituencies of a material object placed in a field of vacuum radiation (in SED representation of quantum vacuum) with definite gradient of energy density.

Consequently, gravitational force thereby arises when an action attempts to prevent the object from performance of the spontaneous (freefall) motion, which is driven by the second law. Accordingly, freefall motion is representation of pure execution of the mandate of the second law for the given conditions while gravitation force is a full hindrance to execution of this mandate. Correspondently, partial hindrances to freefall motion (like fall in a dense medium), i.e. partial deviations from pure execution of the demand of the

3 Given that in point 1 and 2 the system in rest and equilibrium with its surroundings law, meets partial resistance from the system side and consequently generates correspondently less inertial force.

Thus, we can conclude that gravitational field, metric and the vacuum energy density gradient are different manifestations of the same physical field. And, subsequently, a gravitational field can be considered as an effect of the quantum vacuum with diminished ${ }^{4}$ energy density. And, the gravitational potential energy field shall be associated with this vacuum energy density field.

Based on the above deliberation it can be concluded that every material object as immersed in the quantum vacuum is surrounded by a vacuum field, which specified by vacuum energy density (gradient), where the density has a negative gradient towards the center of the field. It can be titled as a proper vacuum field of objects. Hence, an object placed in the field of another larger body undertakes spontaneous motion towards the source of that field, i.e. relocates to locale with lesser vacuum energy density to minimize its energy level. Such, the second law of thermodynamics is accountable for the natural motion of matter in vacuum from an area with greater magnitude of $\rho_{v}$ to an area with lesser $\rho_{v}$.

We introduce here a new term - «diminished» vacuum energy density that requires clarification. The diminished energy density is the energy density of vacuum in the area about material objects, where the magnitude of $\rho_{v}$ is lesser than in the locale infinitely far from all masses, where the impact of the gravitational fields is negligible and where $\rho_{v}$ has its maximal magnitude for the entire region and where $\nabla \rho_{v}=0$.

The role of the proper vacuum field of objects is essential while mechanism of gravitational interaction is considered. For an object positioned far from gravitating masses the proper field is spherically symmetric about the object. However, for the object in to be in the field of other large body the proper field, assumingly, is deformed due to interaction with that global encompassing vacuum field due to superposition of field. So, external action from other objects on the given object is applied through field of other objects and eventually through its own proper vacuum field, which is in fact part of global surroundings modified by matter locally.

The summary of the above is:

(1) It was shown that energy density of vacuum about material objects is diminished, which consists a proper vacuum field of the object; the field has spherically symmetric form when no other masses are in the vicinity;

(2) A notable feature of the proper vacuum field of material objects is distribution of energy density around characterized by the vacuum energy density gradient. It shows that vacuum energy density decreases close to matter and it has certain maximal value in infinity. In other words, boarder of the proper field is specified by the gradient's magnitude tending to zero value in outer border of the field.

4 The term «diminishing» would be more descriptive to use here but we abstain from doing it and giving preference to term «diminished» energy density that was used in past; 
(3) The gradient of proper vacuum field of a massive body determines the energy transfer rate for objects present in the field, to be basis for freefall acceleration.

Matter spontaneously moves from an area with greater $\rho_{v}$ to an area with lesser $\rho_{v}$ to satisfy the second law to minimize its energy and transferring its energy to surrounding vacuum. This transfer of energy as work by falling object mediated by converting part of energy to its more active, kinetic form. This establishes a link between the transfer of energy and motion. The rate of energy transfer defines the acceleration of the falling object.

\section{Analogy Between Microscopic and Macroscopic Systems}

\subsection{Inertia}

Falling objects, as we showed above, are spontaneously moving in gravitational field, minimizing their energy and transferring its energy to surroundings complying with the second law due to the diminished energy density of the surrounding vacuum. On the other hand, as we know, hot objects cool down, i.e. spontaneously minimize their energy dissipating its energy to environment complying with the same law, because of the relative cooler temperature of environ, i.e. reduced energy in surroundings. This gives us a substantial reason to draw an analogy between the thermal behavior of microscopic systems and the inertial motion of macroscopic systems for the exploration of the inertial property of material systems. Due to the equivalence principle, such analogy building shall be applicable for study of both gravitational and inertial behavior of matter. Apparently, in both cases systems' behavior is closely linked with the transfer of energy between systems and their surroundings.

Imagine a simple ${ }^{5}$ closed system, $S$, at rest referred to some fixed system, $K$, and located far from other masses. Consider $S$ being at thermodynamic equilibrium ${ }^{6}$ with its evacuated surroundings. Let's impart energy to $S$ in form of heat, which brings to an increase of its internal energy, $d U$ and its temperature ${ }^{7}$, thereby violating the existing equilibrium. For description of this process we use Crawford's [20] formulation of the first law of thermodynamics ${ }^{8}$ for reversible, quasi-static, or irreversible processes:

$$
d E_{t o t}=Q+A
$$

where, $d E_{\text {tot }}$ is the system's total energy alteration, $Q$ and $A$ are the heat and work added, respectively. The total energy alteration is equal to:

$$
d E_{t o t}=d E_{k}+d E p+d U
$$

where, $d E_{k}$ is the macroscopic kinetic energy, $d E_{p}$ is the

\footnotetext{
5 A system comprised of an ideal gas, where internal energy consists of thermal energy

6 Hereinafter equilibrium

7 For simplification purpose no phase changes involved

8 Herein after the first law
}

macroscopic potential energy and $d U$ represents the internal energy changes. Taking into account that in the process of heat imparting to $S$, from the components of (8) only the internal energy of the system is changing; the energy balance of this process can be expressed as

$$
d U=Q
$$

But, as time passes the system spontaneously dissipates ${ }^{9}$ the received energy to its surroundings ${ }^{10}$ thereby approaching a new state of equilibrium with its surroundings according to the second law.

As we deal with a non-equilibrium thermodynamic process, it is reasonable to express equation (9) in terms of energy transfer rates. So, equation (8) takes the following form:

$$
d U / d t=W_{Q}
$$

where $t$ is time and $W_{Q}$ is the heat transfer rate, $d Q / d t$.

Thus, considering the fact that the heat dissipation leads to the decrease of energy of the system, equation (10) takes the following form:

$$
d U=-\bar{W} d t
$$

where $\bar{W}$ is the rate of heat transfer ${ }^{11}$.

The (instantaneous) natural heat transfer rate, as per (11), is the amount of energy spontaneously released in a shortest period of time, $d t$ and from here $d U=-\int W_{Q} d t$. The term "natural" refers, here, to the fact that the process runs spontaneously complying with the second law.

The above process is manifestation of the natural tendency of the system to minimum energy state confirmed by the second law. At the same time, we affirm that this tendency has to work equally for all components of the total energy of the system expressed in (8) including its internal, potential and kinetic energies since this separation of the total energy to these components is to some extent ${ }^{12}$ conditional.

In this stage we draw an analogy between the behavior of systems undergoing a thermal process and acceleration in regard to their conformity with the second law.

Let us to consider in analogy with $S$, a simple closed system, $S$ ', composed of a material object being at rest ${ }^{13}$ and placed in the same environment, far from gravitational masses, and to be at thermodynamic equilibrium with its surroundings. Consider that some external force hits $S^{\prime}$ causing its acceleration. In this way, $S^{\prime}$, as a thermodynamic system, gains energy throughout the work performed by the external force on it. This work transfers energy from the surroundings to the system resulting in the increase of its kinetic energy for $d E_{k}$ and the violation of the existing equilibrium. In addition,

9 Through radiation and other energy transfer ways

10 It is assumed that given energy is minuscule but surroundings is quite large reservoir

11 As the rate of heat dissipation changes throughout the process depending of temperature gradient hereinafter we imply the averaged value of the rate

12 As the special relativity states that total energy of body is constituted from rest energy and kinetic energy

13 Or state of uniform motion 
this impact has consequence on the surroundings of $S$ modifying vacuum energy density locally (we will consider below). From the analogy between systems $S$ and $S$ ' and the assumed universality of the second law, it is reasonable to assume that the received energy, $d E_{k}$, is subject to natural dissipation (or transfer to the surroundings), as the system seeks to approach a new equilibrium with its surroundings. As we draw an analogy between these two systems, the behavior of $S$ can suggest explanation for the behavior of $S$ '. First, we note that both systems are initially in thermodynamic equilibrium with their surroundings. Therefore:

a) Heat imparted from the system's surroundings to $S$ (or, equally, work performed on it by the surroundings) leads to the increase of its thermal energy that is equal, in this case, to its internal energy indicated by the temperature ${ }^{14}$ increase. This brings it to the breach of the existing equilibrium. To bring the system back to equilibrium, the dissipation of heat from $S$ to its surrounding takes place due to the second law, resulting in the return to temperature of $S$ to temperature of surroundings and thereby approaching a new state of equilibrium.

b) In the microscopic level, impart of energy causes acceleration of motion of constituencies of $S$. However, increased momentum of particles increases energy dissipation to surrounding and brings to speeding down the motion that corresponds to the surroundings' temperature.

c) By analogy, the work done by the surroundings on $S$, accelerates it (regarding reference system $K$ ) and brings to the increase of its kinetic energy violating the existing equilibrium between $S^{\prime}$ and its surroundings. To approach a new equilibrium state due to the second law, the system transfers energy as work to the surroundings (i.e. moving object spends energy for overcoming resistance to its motion), thereby decreasing its kinetic energy; this is observed as decelerating motion. As motion here is assumed to be a consequence of the energy transfer as work by the system, such as for the freefall case, this finally results in halting $S$ ' relative to the reference system $K$, as the energy runs out and the system decelerates approaching new equilibrium, i.e. returns to the initial state of motion (equilibrium).

In fact, the microscopic point of view of $S$ confirms that in case (a) particles of the system behave similarly to the moving object $\left(S^{\prime}\right)$ in case (b). The temperature increase for an ideal gas, for instance, implies an increase of the mean velocity of constituents of matter, i.e. the acceleration of particles to higher velocities taking place as a consequence of heat imparted to the system. In contrast to this, the spontaneous cooling of the substance due to the second law leads to the decrease of the mean speed of the substance's particles, or to the decelerating motion of them through performing work on surroundings via motion of particles and in this way dissipating energy back to surroundings. From here we can conclude that apparently motion is a mechanism of

14 No phase changes considered transferring energy from matter to surroundings ${ }^{15}$.

The above illustration confirms that, in essence, the two cases being compared have significant physical similarity.

Now, let us go back to the study of system $S$ '. In analogy with (11) and considering that $d E_{p}=0$ and $d U=0$ the energy balance for the process of kinetic energy transfer from system $S$ ' to surroundings can be expressed as

$$
d E_{k}=-W^{n} d t
$$

where $W^{n}$, is the rate of kinetic energy transfer and the index $n$ denotes the natural or free character of the energy release process, and $t$ is the time of dissipation.

Equation (12) describes the transfer of kinetic energy by $S^{\prime}$ to its surroundings and it implies that eventually the system comes to rest with respect to the reference system, $K$, as it reaches equilibrium. There is no resistance to its motion, in an ordinary sense, but nonetheless the system will not move incessantly (in the given reference system) as the net energy to be transferred tends to zero ${ }^{16}$, and therefore motion, which was linked to the transfer of energy, will gradually die out. In this context, it is taken that the system's motion takes place in flat, or locally flat, space. Thus, from the analogy we conclude that inertial motion is the effect of the change in energy of body and the associated transfer of energy of it to surrounding medium, i.e. quantum vacuum, which accords to the conclusion drawn from the study of freefall case.

In practice, when the external force fields hindering inertial motion are negligibly weak, the process of kinetic energy transfer as expressed by (12) lasts sufficiently long that is perceived as endless uniform motion. It is due to the very small magnitude of the natural energy transfer rate, as in a thermal process, when a very slow cooling rate gives a very long heat dissipation time and a very small and almost constant rate of heat dissipation.

Let us go back to system $S$ being in thermodynamic equilibrium with its surroundings. Imparting heat, $d Q$, to the system breaches the existing equilibrium between $S$ and its surroundings, and brings about heat dissipation from $S$ to its surroundings in order to approach a new equilibrium. This way, the system spontaneously changes its internal energy level from $U_{1}$ at temperature $T_{1}$ to $U_{2}$ at $T_{2}$, where $T_{1}>T_{2}$. According to the first law of thermodynamics the system can realize this transition by transferring heat $d Q$ or by performing work, $A$, on surroundings. The process can be formulated as

$$
d U=U_{1}-U_{2}=d Q=A .
$$

We ought to note here that it is essential to the thermodynamics of energy transfer processes that this process occurs at a finite rate. Consider a purposeful speed-up of the

15 Thus, an object thrown up does not convert its energy to potential form, how it is assumed conventionally, instead it spends kinetic energy for motion, translation the object to a new locale with different (higher) field potential and the object possesses potential energy just because of presence in the field location with different parameters.

16 Assuming that the surroundings is homogeneous and extended to enough large area. 
latter process of heat transfer from $S$ to its surroundings in order to reach equilibrium earlier, i.e. to make the heat transfer process faster than its natural, free rate set by the second law for the given conditions. It is important to note that we talk about the transfer of the amount of heat, which according to the second law is subject to dissipation from $S$ to its environment in order to reach equilibrium and no more than that. In order to accomplish this, there is a need to remove heat from the system ${ }^{17}$ faster than it would spontaneously occur in the natural way as per (13).

The energy balance for this process according to the first law (1) can be formulated as

$$
d U=-\left(W^{Q n} d t+d Q\right)
$$

where, $W^{Q n}$ is the average natural energy dissipation rate, $d Q$ is the heat removed from the system through work in the shortest period of time, and the index $n$ denotes the natural, spontaneous character of the heat transfer process, i.e. the dissipation process. Equation (14) can be read as: the decrease in the internal energy of the system (in a short period of time, $d t$ ) equals to the heat spontaneously dissipated by the system and the heat removed from it by applying external work.

On the other hand and in the ideal case, the heat is being removed, $d Q$, is equal to the work that the system performed on surroundings to counter the forced change in the operation of energy transfer from the system to its surroundings, which is denoted here by $A$.

Let us consider a hot object, which is spontaneously dissipating heat to its relatively cooler environment. In order to make the cooling process to occur faster, or to accelerate the heat transfer from it to the surroundings, a heat pump can be mounted on the system. Operating such a pump for removing energy at a faster rate than the system is ready to spontaneously release without any thermodynamic operation, requires a supply of external energy. Therefore, the release of additional amount of energy in the same period of time is possible through performing thermodynamic work.

With the above considerations expression (14) can be transformed into the following form:

$$
d U=-\left(W^{Q n} d t+A\right)
$$

where, $A$ is the work performed by $S$ equivalent to the heat $d Q$.

Transformation of (15) with consideration of the definition of the energy transfer rate expressed in equation (11), and introduction of the rate of work transfer, or power, $N=A / d t$, yields

$$
W^{Q}=W^{Q n}+N
$$

where, $W^{Q}$ is the current heat transfer rate $\left(W^{Q}=d U / d t\right)$. The last expression shows that total rate of energy transfer from the system to its surroundings is equal to the sum of the spontaneous energy dissipation rate, $W^{n}$, plus the work transfer rate for work performed by the system on

17 In this stage we intentionally consider only active actions on the system rather than actions on the surroundings such as cooling down the surroundings surroundings given by $N$.

Now, imagine an analogous case for system $S$, where the (kinetic) energy transfer process from it to its surroundings is forced to speed up. Such action (operation) on the system interferes with the free motion of the system and violates the natural fulfillment of the second law. This can be realized, for instance, through providing resistance to the motion of the system, thereby requiring additional expend of kinetic energy by the system to do the work needed to overcome this resistance to its motion. This results in the faster transfer of kinetic energy from the system ${ }^{18}$. The energy balance for this process is represented by:

$$
d E_{k}=-\left(W^{n} d t+A^{\prime}\right),
$$

where, $W^{n}$, is the rate of free kinetic energy transfer and $A$, is work performed by the system to overcome the resistance to its motion. A good demonstration of process (16) would be a body undergoing inertial motion that meets resistance created by some external force, for example, resistance due to drag. In order to overcome the resistance, the body performs work and in this way expends more of its kinetic energy per unit of time; we say in such case that the system transfers its energy as work. Consequently, the system transfers kinetic energy more quickly than in the case of free motion, which gives a larger absolute magnitude of $d E_{k} / d t$, i.e. it causes higher deceleration of $S^{\prime}$. This exerted hindrance force is balanced by the system's resistance to the forced change of its kinetic energy transfer rate that alters resulting rate making it different (higher) than the rate set by the second law, which we specify as spontaneous or free energy transfer in the given condition. In other words, an external hindrance applied to the inertial motion of $S$ ' is countered by the system's internally generated resistance to maintain the rate of the spontaneous kinetic energy transfer as established by the second law. For example, when a car undergoing run-out suddenly applies the brakes, the car resists the introduced change of its energy release pattern by loading the brakes and performing work on environment. A simple transformation of (17) with replacement of $A^{\prime}=N^{\prime} d t$ better describes this process:

$$
d E_{k} / d t=-\left(W^{n}+N^{\prime}\right),
$$

where, $N^{\prime}$ is the rate of energy transfer additionally extracted from the system (by doing work on surroundings) to overcome resistance to its motion. Equation (18) shows that the absolute magnitude of the time derivative of kinetic energy, $d E_{k} / d t$, increases due to the imposed work performed by the system that depicted here by $N^{\prime}$. In other words, it illustrates that an introduced hindrance increases the rate of kinetic energy outflow causing additional deceleration of the system.

If a system is spontaneously transferring energy to its surroundings at a certain rate that complies with the second law, any forced operation to accelerate this process, even if it aimed in the same direction of energy transfer, meets resistance from the system that is trying to maintain the

18 In the general case, spending of energy by the system means transferring energy to surroundings 
established energy transfer rate. Such action requires an expenditure of energy, as the system generates resistance to the imposed energy transfer from it to its surroundings at a rate higher than the rate of free ${ }^{19}$ energy release for the given conditions and established by the second law. Generation of resistance is explained by Le Chatelier's principle in its general form requiring that, when there is an external constraint on a system, a behavioral shift in the system occurs so as to annul the effect of that change. For the case under our consideration, it means that the system opposes the action aimed to boost the transfer of heat from it above its spontaneous rate, as set by the second law. This emerged resistance is balanced by the external work applied to the system and conventionally identified as effect of inertia.

Yet, imagine the opposite case, when for the above system $S$ the heat transfer rate is forcedly decreased relative to its natural rate set by the second law. Let the system to be in the process of dissipating heat to its surroundings in order to reach a new equilibrium with the surroundings. This can be achieved by decreasing the net energy outflow from $S$ to its surroundings. Let us use for this purpose again consider a heat pump that working in a reverse mode, i.e. supplying heat to the system that reduces the net outflow of heat from the system. The operation will meet resistance proportional to the rate of the organized heat inflow (supply) as it changes the natural energy transfer pattern established by the second law. Therefore, in this case the heat pump needs external energy supply, as work or heat, for its operation. Emergence of the resistance again aligns with Le Chatelier's principle. This supply of heat leads to the reduction of the total heat transfer rate from $S$ to its surroundings. The energy balance for this case can be expressed as

$$
d U=-\left(W^{n} d t-A\right) .
$$

This equation states that for the system naturally approaching equilibrium through the transfer of heat to environment conforming to the second law, the heat transfer rate can be reduced by performing work, $A$, on the system. Expression (19) coupled with consideration of the heat dissipation rate (11) and $N=A / d t$ takes the following form:

$$
\mathrm{W}=\mathrm{W}^{\mathrm{n}}-\mathrm{N} \text {. }
$$

This last derivation shows that the current magnitude of $W$ is decreasing the natural energy dissipation rate due to work, $A$, performed on the system with transfer rate $N$. And, this operation, directed to change the natural pattern of energy flow, also meets resistance generated by the system.

Now, imagine an analogous case for $S^{\prime}$, in which, we consider the possibility of slowing down the process of kinetic energy transfer from $S$ ' to its surroundings, that is decreasing

\footnotetext{
19 Ideally, the term "free" cannot accommodate the case when an object is immersed in a cooler medium, for instance air, as an ambient medium utilizes a convection mechanism that speeds up cooling process and the body spends extra energy for this work. In this sense an ideal case for setting free- natural energy transfer conditions would be a vacuum, where the system under the study can transfer heat by both radiation and conduction
}

the energy transfer rate that therefore leads to a prolonging the run-out motion of $S^{\prime}$. For the moving systems this can be done by applying an external force performing work on the system in the direction of its run-out motion.

In analogy with the above analyses the energy balance for this process can be written as:

$$
d E_{k}=-\left(W^{n} d t-A^{\prime}\right) .
$$

The above equation shows that the positive work, $A$ ', done on the moving system $S$ ' to slow down its energy transfer rate to its surroundings, increases kinetic energy of the system. This means ensuring additional energy inflow from the surroundings to the system that can compensate for the energy transferred by the system as work. In fact, it implies decreasing magnitude of deceleration of $S^{\prime}$.

As equation (21) shows, depending on the magnitude of $A$ ' relative to $W^{n}$ ' $d t$, there can be cases of full or partial replenishment of the transferred kinetic energy. It means that even for the case of maintaining constant speed of moving bodies there is a need to supply energy to the system (case $W^{n}$ ' $d t=A^{\prime}, A^{\prime}>0$ and $d E_{k}=0$ ). This energy is brought to the system through performing work on $S^{\prime}$ to overcome the resistance arising from the change of the energy transfer rate, that is different from the natural energy transfer rate as per the second law for given conditions. The appearance of this resistance explains the system's resistance to the accelerating force. The system's acceleration is defined by the balance between the externally imposed force and the internally generated resistance. This, however, does not cover the ideal case of uniform motion.

Imagine an object thrown in space far from gravitating masses that is experiencing run-out motion. This motion, according to our conclusion, is a direct consequence of the transfer of (kinetic) energy from the object to its surroundings as work and, therefore, with the expiration of the energy the body is decelerating. In order to slow down the energy transfer, i.e. to prolong the run-out motion of the body there is a need, as for system $S$, to ensure a decrease of net energy outflow (transfer) rate to surrounding, which is equivalent to providing an inflow of kinetic energy to the body ${ }^{20}$. This can be done by supplying add-on kinetic energy through the application of a force on the body adding acceleration (or reducing its deceleration) in the direction of its current motion and thereby performing work on it, or by reducing the drag on its current motion. Introducing such external action to change the energy transfer rate set by the second law meets resistance generated by the system and therefore requires a balancing force performing work on the system.

From expression (21) we derive: if a body is accelerating from rest, assuming that the energy dissipation is negligibly small, yet $\left(W^{n} \rightarrow 0\right)$, it yields $d E_{k}=A^{\prime}$ leading to $d E_{k} / d t>0$, which means that rate of energy transfer from the system to its surroundings is negative, i.e. this is the case when incoming energy to the system exceeds the energy loss due to the energy

20 We note that we only consider operations done on the system but not on its surroundings 
transfer. We have the same result when $W^{n}, d t<A$, too. As a particular case of this process there is the situation, when $W^{n} d t$ $=A$ ' leading to $d E_{k}=0$, meaning that even for maintaining kinetic energy of a system (or constant velocity in flat space) there is a need to transfer some amount of energy to the system to compensate for amount of energy transferred as work.

Thus, the above analyses show that both systems, $S$ and $S$, respond identically to the external operation aiming at changing the spontaneous process of energy exchange between a system and its surroundings regulated by the second law. So, in general, mechanical behavior of microscopic and macroscopic systems is governed by the same laws.

At this point we give a general form to the energy balance equations for a moving body (18-20) drawn from the analogy with thermodynamic system:

$$
d E_{k} / d t=-W^{n} \pm N^{\prime}
$$

where, $N^{\prime}=A^{\prime} / d t$ is power of external work on the system.

Thus, from equation (22) we can specify the following cases:

A) An acceleration case is defined when $N^{\prime}>0,\left|N^{\prime}\right|>\left|W^{n}\right|$ and consequently $d E_{k} / d t>0$. As a particular case of acceleration we have acceleration from rest, when $N^{\prime}>0$, $W^{n}=0$ and $d E_{k} / d t=N^{\prime}$ so that the external work done on the system leads to a positive change (increase) of kinetic energy of the system;

B) A deceleration case is defined when $N^{\prime}<0$, i.e. a drag applied to motion; consequently $d E_{k} / d t<0$, or when $N^{\prime}>0$ and $\left|N^{\prime}\right|<\left|W^{n}\right|$, i.e. the external action does not fully compensate energy loss due to its transfer to surroundings. Consequently $d E_{k} / d t<0$; If in this setting we have $N^{\prime}>0$ and $\left|N^{\prime}\right|>\left|W^{n}\right|$, then we get again acceleration case, (A);

C) Pure inertial motion as a particular case of deceleration is defined when $N^{\prime}=0$, and consequently $d E_{k} / d t<0$;

D)An idealized case of uniform motion (it also implies state of rest) is realized when the system at equilibrium with its surroundings, where net energy transfer is zero and hence $N^{\prime}=W^{n}=0$ and $E_{k}=$ const.

So, the above analysis fully elucidates that inertia is a consequence of changes in the energy of the system. The related inertial motion is due to the system's spontaneous energy transfer as work to its surroundings at a rate established by the second law for the given conditions (resulting in motion) when the system approaches equilibrium. Any action directed to alter this established natural rate of (kinetic) energy transfer meets resistance from the system as it necessarily violates the requirements of the second law.

\subsection{Gravitation}

At this point we investigate gravity using the laws of thermodynamics and showing that equation (22) can be also applied as a description of gravitational freefall motion. Consider case (A) of (22), it can be obtained if we apply equations (1) and (2) to the analysis of energy transfer for a system undergoing freefall. The final result of analysis will have the following form:

$$
d E_{k} / d t=-W^{n} \pm N^{\prime}+N_{G}
$$

where $N_{G}$ is the power of external work exerted by the gravitational field causing acceleration of the body in freefall and is equal to $m g(d r / d t)$.

A) The pure freefall case, when falling object has no hindrance to its free motion and which corresponds to the freefall acceleration, $g$. For this case $N^{\prime}=0, W^{n}=0$ and $N_{G}>0$, so consequently equation (23) takes this form: $d E_{k f f} d t=N_{G}$. Here, $E_{k f f}$ is kinetic energy of object corresponding to its freefalling case. It is a particular case where solely $N_{G}$ generates motion with positive $d E_{k f f} d t$ corresponding $g$;

B) The case of falling with an acceleration higher than $g$, is a case when a freefalling object is additionally accelerated by external power $N^{\prime}>0$ (work performed on the system), $W^{n}=0$ and $N_{G}>0$ consequently $d E_{k} / d t$ increases $\left(d E_{k} / d t>d E_{k f f} / d t\right)$;

C) The case of falling with an acceleration smaller than $g$, is motion of an object in a medium with drag when $N^{\prime}<0$ (the system has to perform work on its surroundings), $\left|W^{n}\right|>0$ and $N_{G}>0$, and so consequently $d E_{k} / d t$ decreases $\left(d E_{k} / d t<d E_{k f f} / d t\right)$. At a certain condition when $\left|N^{\prime}+W^{n}\right|>N_{G}$ it can result in the deceleration of the object: $d E_{k} / d t<0$.

The freefall process has some features that are useful to explore here. As is known from the freefall experiment, no device mounted on the falling body can detect its acceleration, whilst the rate of velocity change of the falling body to an external stationary observer clearly shows acceleration. It is because gravitational freefall is a process of free, or natural, kinetic energy change, when the second law is purely followed $^{21}$ and therefore the acceleration is not detectable. This is the case when both the system under study and a test instrument strictly meet requirement of the second law, and in this way they cause no resistance to each other, hence generating no indication of acceleration on the measuring device. The implication of the last sentence for inertial motion $^{22}$, in general, is that the natural kinetic energy change in the case of inertial motion with $d E / d t \neq 0$ is also not detectable. This is true, even if an external observer kinematically detects a change in the velocity. Thus, the latter conclusion supports our initial finding that inertial motion is, in fact, a form of deceleration, i.e. a form of spontaneous motion linked with an energy release from the system where no device mounted on the object undergoing inertial motion detects acceleration.

\subsection{The Equivalence Principle}

We defined that gravity and inertia can be explained by thermodynamic principles, where gravity is a consequence of the decrease in the energy (density) of surroundings of a system and acceleration is an effect of the increase in energy of system itself. These findings, if match up, allow us to

21 It means that the process is not interfered with by external factors but is performed purely as demanded by the second law of thermodynamics both by the system and parts of measuring device

22 That is equivalent to the freefall case 
validate our conclusions made above and provide a deep insight into understanding the intrinsic nature of this principal.

a) Consider system $S$ (considered above) to be in thermodynamic equilibrium with its surroundings, now imagine that energy imparted to $S$ at certain rate (as heat or as work) to bring up its internal energy. This operation violates equilibrium and does not comply with the second law, which works to establish equilibrium. As it does not comply with the second law performing this thermodynamic operation requires energy expend.

b) Consider, again, $S$ in thermodynamic equilibrium with its surroundings, but now think of the case when somehow energy of surrounding is being diminished, this operation immediately entails energy release process from $S$ to surroundings to maintain equilibrium of the system with its surroundings due to the second law. To avoid this outcome and preserve energy of $S$ there is need for imparting energy (as heat or as work) to $S$ at certain rate. This operation does not comply with the second law, which tends to establish equilibrium, i.e. decrease energy of $S$ by the process of the energy release from it to surroundings. Consequently, the conservation of energy for $S$ in this condition requires, again, energy expend.

c) Imagine, in analogy, systems $S$ ' (also considered above) to be in mechanical equilibrium (with its surroundings), imagine that a force applied to $S$ ' that accelerates it increasing its kinetic energy. This impact violates existing equilibrium and does not comply with the second law and consequently meets resistance to its performing, which is known as inertia.

d) Imagine $S$ ' to be in mechanical equilibrium (with its surroundings), think of the case when somehow energy density of surrounding is being diminished. This causes the system to minimize its energy as demanded by the second law. For gravity, as we see above, there appears energy density gradient around the source of surroundings' energy density decrease, which causes spontaneous motion of $S^{\prime}$ to locale with less energy density with following kinetic energy generation and the energy transfer it to surroundings. However, in order to maintaining stationary state of the system (which is equal to preserving energy of $S^{\prime}$ ) there is need for performing work on $S$ ' to hold it from freefall motion in the field imparting energy (as work) to $S^{\prime}$ at certain rate. This action (operation) to prevent the material object from freefall, i.e. prevents the system to fulfill the second law that consequently meets resistance, which is known as gravity phenomenon.

The response behavior of a system to the effect of its acceleration, i.e. increasing its kinetic energy, is identical to the response behavior of it to the effect of holding the system from freefall in the gravitational field, i.e. preventing system from spontaneous minimization of its energy in the surroundings with decreasing energy density or in surroundings with gradient of energy density.

As we see, mechanical behavior of a system consistent with typical thermodynamic behavior of systems when energy of the systems is increasing or energy of surroundings is decreasing.

In generalized form the equivalence principle upholds that for a system to be in equilibrium with its surroundings, energetic implication of both operations: (a) the increasing energy of the system (while energy of surroundings is unchanged) and (b) the conserving the system's energy when energy of the surroundings is decreasing, is equivalent. Accordingly, mechanical behavior of systems is identical, for both acceleration and the rest state in gravitational field. This clarifies why all material objects responding identically to gravitation and inertia.

Based on above considerations we give the following is definition of the equivalence principle: for the system that prior to that was in equilibrium with its surroundings the increase in energy of a system and the decrease of energy of its surroundings are equivalent operations since both have identical implications for the system related with consequences of the second law.

\section{Implication of the Proper Vacuum Field for Explanation of Inertia's Mechanism}

This work has no aim to study mechanism of gravity and inertia based on above findings. However, it gives very tentative projection of how those mechanisms to be if grounded on this work results.

It was established above that for un-accelerated material system to be positioned far from gravitating masses the proper vacuum field is spherically symmetric around the system. When the system placed in the field of other larger object (gravitation) the field of the considering object is deformed with clear asymmetry around the object due to local changed energy density of the field and to be cause of the gravitational force. In case of acceleration such deformation of the proper field is estimated due to the localized transfer of energy between the system and its surrounding vacuum accompanied with motion of the object that will be again cause of appearance of the inertial force.

\section{Discussion}

The idea that gravity is an emergent phenomenon has a long history although has not yet been widely recognized. There were many suggestive analogies drawn between gravity and thermodynamics supporting this concept. However, only a few recent efforts promote this idea, like one that undertaken by Verlinde [7] and showing the existence of an entropic force from the gradient of the entropy through the holographic approach, were quite successful.

This paper likewise suggests that gravity and inertia are emergent, but the paper follows a conceptually new approach to show it. While being similar in spirit to Verlinde's work, our effort emphasizes that gravity can emerge from the gradient of surroundings' energy density. There exists a link between the change in entropy and the change in energy of a system and its 
surroundings that relates this work to the above mentioned one.

Newton's gravity is purely phenomenological (descriptive) in its nature, whereas general relativity explains gravity as a curvature of space but limiting itself to the geometrical properties of it. Entropic gravity, which is currently undergoing recognition, defines gravity as phenomenon related to the physical properties of surrounding medium, entropy. In this work we show that gravity and inertia can be defined by the energetic properties of the surrounding medium. In this way, this work relies on physical properties of medium providing solid alternative to the dominating geometrical interpretation of space. Herein, it is very notable that this study employs thermodynamic principles. We note that similar result that gravity can actually be explained as effect of changes in the energy density of quantum vacuum obtained also in [18] from condensed matter standpoints.

The results of this paper suggest that gravity and inertia are emergent phenomena. If the gravity and inertia can actually be explained as effect of changes in the energy density of vacuum, this should have important implications for many areas in which those phenomena play a dominant role. It is especially interesting to examine the consequences for many physic occurrences and cosmology. Such, the way how the redshift (blueshift) and bending light arise from the vacuum energy density gradients, investigation of the nature of black holes or how the vacuum energy density alteration contributes to the universe expansion could bring about new insights.

Results of this work suggest that density of energy of the vacuum fields about material objects is diminished and its gradient indicating that the density decreasing toward the object. It is logical to assume that massive objects more decrease the energy density of vacuum than smaller objects. Subsequently, we can assume that for a very massive celestial body the drop of vacuum energy density, theoretically, may reach in certain proximity to the center of the body its critical value when vacuum fields cannot sustain propagation of electromagnetic waves and this, most likely, corresponds to the event horizon.

There were many proposals that gravity is emergent phenomenon, see [6] for a review of these. But this work in unique way uses thermodynamic principles to describe mechanical behavior of thermodynamic, material systems immersed in quantum vacuum represented as energy reservoir. We derived Newton's second law and discovered the origin of inertial force in vacuum context using thermodynamic principles. We identified a cause of gravity and inertia that turns out to be differences in energy of a system and surrounding medium driven by the second law which causes motion.

Here, we would like to particularly mention Clerk Maxwell hypothesis that corroborate results and approach of our work. In his time he made a profound comment on the nature of gravitation [21] saying that the attraction effect of gravity is probably the result of diminishing energy in the surrounding medium. He further assumed that the energy of the medium must be somehow decreased by the presence of the bodies in it. And, this work fully confirms that concept and finds corroboration though Casimir effect.

For this study we employ a model in which behavior of a material system immersed in the quantum vacuum energy reservoir is analyzed using general thermodynamic deliberations. Use of vacuum context is encouraged by the concept of the induced gravity that proposed by Sakharov [8] and Gamov's idea that Dirac vacuum has to exhibit itself through gravitation [22]. Additionally, we inspired by the trend in scientific literature that ascribes to the energy of quantum vacuum fields the role of dark energy in the universe.

But what is particularly unique about this inquiry is that it studies gravitation and inertia separately providing independent thermodynamic explanations of those phenomena, i.e. without exploiting the equivalence principle for the extrapolation of the result obtained for one phenomenon to the other phenomenon. Consequently, such independent reasoning of those phenomena allowed deriving a unique thermodynamic definition for the equivalence principle, which also became additional means of the validation of this work. As result it allowed us to derive a unique, thermodynamic definition of the equivalence principle that reveals its real, as we suppose, nature. It states that the reaction of a system residing in thermodynamic equilibrium with its surroundings is identical to the violation of the equilibrium either due to (a) the change (decrease) in energy of surroundings (corresponding to gravitation phenomena) or due to (b) the adequate change (increase) in the energy of the system (corresponding to acceleration, inertia case). Both cases equally result in triggering the tendency of the system to minimize its energy state and the related energy transfer from the system to its surroundings as work - putting object to motion in compliance with the second law.

It seems it is not consistent that the gradient of energy density of surroundings turns out to be key for gravity but the rate of energy transfer is key for inertia case (how we conclude in this work) whereas these two phenomena linked through the equivalence principle. However, there is a hidden coherence between these two factors that can explains this: the rate of energy transfer, for inertia, (for the given conditions) defines acceleration, when identically the gradient of energy density of surroundings, for gravitation, determines the rate of energy transfer for a freefalling system, which in its turn defines acceleration of freefall motion. That is finally both define acceleration of the system.

We draw an analogy between behavior of microscopic and macroscopic systems demonstrating the key role of principles of thermodynamics in explanation of respective behavior in both scales and showing the emergent nature of gravity and inertia. Different microscopic scale appearances driven by the second law are known to us (as osmosis, convection, elasticity etc.) that appear in interference with other properties of gases and fluids in atomic and molecular levels and in such cases understanding that gravity is also consequence of the second law is quite challenging, in particular when the same principle sometimes acts in counter directions. Gravitation in microscopic level is, often in such cases, untraceable compare to other phenomena since it is very week in that scale. On 
macroscopic scale, however, the total effect of collected matter gives rise to the clear manifestation of the phenomenon known to us as «gravitation», emergence of which becomes evident.

It is a key finding of our study that density of energy of surrounding medium around material objects has the diminishing pattern resulting in appearance of attraction effect that, very tentatively, finds corroboration though Casimir effect. It also explains why clocks run slower near massive objects concluding that the energy deficiency in vacuum slows the run of processes in subatomic level analogical to what we usually observe for thermal processes when internal energy of surroundings is decreased, similar argument presented in [23].

This work shows that gravity and inertia are emergent phenomena and the second law has the key role in this. The above suggests that the second law, which is held accountable for the governing transfer (or motion) of energy in nature, affirming that heat spontaneously moves from hot to cold place, is accountable for governing motion of masses in the universe, supporting that material objects spontaneously move to the area with lesser energy density of surrounding medium. It is very important point as it illustrates how the mass-energy equivalence confirms and further extends universality of the second law suggesting that motion of matter in the universe is also governed by the same law.

If light deviates from a straight line in gravitational field and this work affirms that a quantum vacuum with variable density is equivalent to it, then an experiment on vacuum with gradient of its energy density shall prove or refute this claim. For such experiment there is need for two as much as possible large ideally flat plates positioned close to each other (on smallest distance comparable with Casimir effect type experiment), but with certain acceptable slope relative each other in order to maintain considerable gradient of vacuum energy density between plates. And, if the proposal is true, the deviation from straight line propagation shall be detected for a light ray passing between the plates.

The results of this study suggest solutions to some important problems of physics and cosmology. Discovery of cosmic microwave background showed that early universe had inhomogeneities in the primordial background energy fields. Implication of our study suggests that those regions of space with lesser energy density, acting as primordial gravitational fields trapped diluted matter holding them together like pools, and therefore play the main role in the structure formation. This is more plausible argument than the accepted view that formation started due to the gravitational attraction of the slightly denser regions of matter in the case when matter was distributed almost uniformly, in that stage. It is because, in the latter case, there still can be questioned why first matter particles were unevenly distributed.

There is a recognized view that gravitational systems tend towards non-even distribution of mass and energy across a space region, which appears does not harmonize with the spirit of the second law ${ }^{23}$ and holding it responsible for

23 To be responsible for even (uniform) distribution of matter gravitational interaction in this study. This view seems to challenge outcomes of this work as it affirms that even for gravitational systems the second law works according to its original mandate, i.e. to even out energy and mass distribution in the area. The matter is that the classical, conventional approach in physics exclusively focuses on energy of matter. However, inclusion of background energy (of quantum vacuum) as an all-embracing energy reservoir to the analyses changes that conservative view. In this way, we think that our approach suggests that physical systems all the time (including in gravitation case) tend to even distribution of mass and energy (but not mass alone) throughout entire universe as confirmed by observations. Consequently, space area with massive concentration of matter suffers from energy deficiency of vacuum and vice versa (matter tends to quit area of high vacuum energy), which is in full accordance with the mass-energy equivalence. This implies that the mass and energy distribution role of the law may not be evident in thermodynamic analyses where consideration is limited by solely mass or solely energy analysis as it is conventionally done.

The above two points highlight a concept that the second law has key role in the distribution of mass and energy in the universe and consequently in explanation of the universe expansion. Thus, the above conjunction of two fundamental principles of nature provides additional support to the results of this work and enhances exploration value of it.

We admit that the work we present is part of a new endeavor by its nature and therefore its results pretend to be heuristic, nevertheless this should not reduce value of this pioneering work.

This work possibly will face a challenge related with conventional application of thermodynamic principles. Conservatively, we used to allow consideration of transfer of energy to surroundings as dissipation of energy. However, energy transfer from imponderable, ambient system like vacuum surroundings to a system composed with ponderable matter does not accord with this conventional view, especially if is related with mechanical behavior of the system. For instance, acceleration, in conventional application, implies energy transfer as a work from another system, whereas in thus work we explain this transfer of energy from surrounding medium to the system due to an external work to make this transfer to happen.

\section{Conclusion}

Thus, results of this work affirm that gravity and inertia are emergent phenomena and the second law has the key role in this. The study clearly shows that systems undergoing inertia and gravity do follow the same - generic pattern of behavior that wide-spread in nature and driven by the second law. This implies that gravitation is not fundamental interaction, but typical manifestation of systems' behavior driven by the second law.

The main argument of this paper is that classical thermodynamics provides a natural and reasonable 
explanation of emergence of gravity and inertia. What is very important, it makes such argument out of conventional thermodynamic consideration of interaction between material objects and quantum vacuum, as a system and surrounding.

\section{Acknowledgements}

We would like to express our gratitude to Ph.D. Eric Weiss for support in preparation of this article.

\section{References}

[1] J. D. Bekenstein, Black holes and entropy, Phys. Rev. D 7, 2333 (1973).

[2] S. W. Hawking, Particle Creation By Black Holes, Commun. Math. Phys. 43, 199 (1975) [Erratum-ibid. 46, 206 (1976)].

[3] J. M. Bardeen, B. Carter and S. W. Hawking, The Four laws of black hole mechanics, Commun. Math. Phys. 31, 161 (1973).

[4] T. Jacobson, Thermodynamics of space-time: The Einstein equation of state, Phys. Rev. Lett. 75, 1260 (1995) [arXiv: gr-qc/9504004].

[5] T. Padmanabhan, Classical and quantum thermodynamics of horizons in spherically symmetric spacetimes, Class. Quant. Grav. 19, 5387 (2002) [arXiv: gr-qc/0204019].

[6] T. Padmanabhan, Thermodynamical Aspects of Gravity: New insights, arXiv: 0911.5004 [gr-qc].

[7] E. P. Verlinde. On the Origin of Gravity and the Laws of Newton, JHEP 1104 (2011) 029 [arXiv: 1001.0785 [hep-th]].

[8] A. D. Sakharov, Vacuum quantum fluctuations in curved space and the theory of gravitation, Sov. Phys. Dokl. 12, 1040 (1968) [Dokl. Akad. Nauk Ser. Fiz. 177, 70 (1967 SOPUA, 34, 394.1991 GRGVA, 32, 365-367.2000)].

[9] E. P. Verlinde, Emergent Gravity and the Dark Universe, SciPost Phys. 2, 016 (2017) [arXiv: 1611.02269v2 [hep-th]].

[10] R. G. Cai, L. M. Cao and Y. P. Hu, Hawking Radiation of Apparent Horizon in a FRW Universe, Class. Quant. Grav. 26, 155018 (2009) [arXiv: 0809.1554 [hep-th]].
[11] R. G. Cai, L. M. Cao and Y. P. Hu, Corrected Entropy-Area Relation and Modified Friedmann Equations, JHEP 0808:090, 2008 [arXiv: 0807.1232 [hep-th]].

[12] R. Cai, L. Cao, N. Ohta, Friedmann equations from entropic force, Phys. Rev. D 81 (2010) 061501.

[13] Y. Cai, E. N. Saridakis, Inflation in entropic cosmology: Primordial perturbations and non-Gaussianities, Phys. Lett. B 697 (2011) 280.

[14] Y. Cai, J. Liu, H. Li, Entropic cosmology: a unified model of inflation and late-time acceleration, Phys. Lett. B 690 (2010) 213.

[15] D. A. Easson, P. H. Frampton, G. F. Smoot, Entropic accelerating universe, Phys. Lett. B 696 (2011) 273.

[16] Péter Ván and Sumiyoshi Abe, Emergence of modified Newtonian gravity from thermodynamics ArXiv: 1912.00252 (cond-mat).

[17] L. M. Caligiuri, A. Sorli, "Relativistic energy and mass originate from homogeneity of space and time and from Quantum Vacuum energy density", American Journal of Modern Physics. Vol. 3, No. 2, 2014, pp. 51-59. doi: 10.11648/j.ajmp.20140302.14.

[18] L. M. Caligiuri, A. S. Sorli, Gravity originates from variable energy density of quantum vacuum, American Journal of Mod. Phys., 2014; 3 (3): 118-128.

[19] M. Planck, On the dynamics of moving system, presented at the meeting of 13 June 1907 (Zur Dynamil bewegter Systeme, Sitz. ber. Preuss. Akad. Wiss., pp. 542-570 Berlin).

[20] Crawford, F. H. (1963). Heat, Thermodynamics, and Statistical Physics, Rupert Hart-Davis, London, Harcourt, Brace \& World, Inc..

[21] C. M. Maxwell, A dynamical theory of the electromagnetic field, Philosophical Transactions of the Royal Society, vol. 155, part IV, 1865.

[22] A. D. Dolgov, Y. B. Zeldovich, M. B. Sajin, Cosmology of the early universe, Moscow State University press, 1988; ISBN 5-211-00108-7.

[23] A. S. Sorli, Mass-Energy Equivalence Extension onto a Superfluid Quantum Vacuum, Journal Nature Phys., vol. 9, 11737 (2019). 